\title{
Future usage of quasi-infinite depleted uranium target (BURAN) for benchmark studies
}

\section{P. Tichý1 , M. Suchopár}

\section{for collaboration Energy plus Transmutation of RAW}

Nuclear Physics Institute of the ASCR, v. v. $i$.

Řež 130, 25068 Řež, Czech Republic

Faculty of Nuclear Sciences and Physical Engineering, Czech Technical University in Prague

Břehová 7, 11519 Prague 1, Czech Republic

E-mail: tichylujf.cas.cz, suchoparaujf.cas.cz

The development of advanced nuclear systems needs reliable and accurate simulation codes. Significant necessity are benchmarks of Monte Carlo simulation programs (e.g. MCNPX), which are important for studies of neutron production, transport and transmutation of radioactive materials by produced neutrons. Experiments with various setups consisting of lead, natural uranium and graphite irradiated by relativistic protons and deuterons are used for such purposes at JINR Dubna. Experiments with new setup called „Buran“ should start soon. Preliminary calculations (in MCNPX code) of proton and deuteron beam transport through this setup and neutron production and fluxes in different places of such setup have been made. Representative results are shown and discussed.

XXII International Baldin Seminar on High Energy Physics Problems

15-20 September 2014

JINR, Dubna, Russia

\footnotetext{
${ }^{1}$ Speaker

(C) Copyright owned by the author(s) under the terms of the Creative Commons Attribution-NonCommercial-ShareAlike Licence.
} 


\section{Introduction}

Energy and Transmutation of Radioactive Waste (E\&T RAW) is a collaboration formed by members from 15 countries that is involved in study of neutron production and transport in model ADS (Accelerator Driven Systems) setups. The neutrons are created by spallation reactions of relativistic protons and deuterons with setup target and their consequent multiplication in setup blanket. Experiments with these setups are performed in JINR Dubna and up to now four such setups has been made and irradiated. They are: Gamma-2, Gamma-3, Energy plus Transmutation and Quinta. A new setup called Buran is now being prepared and some neutron behavior predictions have been calculated.

\section{Materials and methods}

\subsection{Experimental setup}

Fundamental part of the Buran setup (see fig. 1) is huge cylinder made from depleted uranium $\left(0.3 \%{ }^{235} \mathrm{U}\right)$ with longitudinal distance of $1000 \mathrm{~mm}$ and $1200 \mathrm{~mm}$ in diameter. The cylinder is surrounded by $100 \mathrm{~mm}$ steel covering.

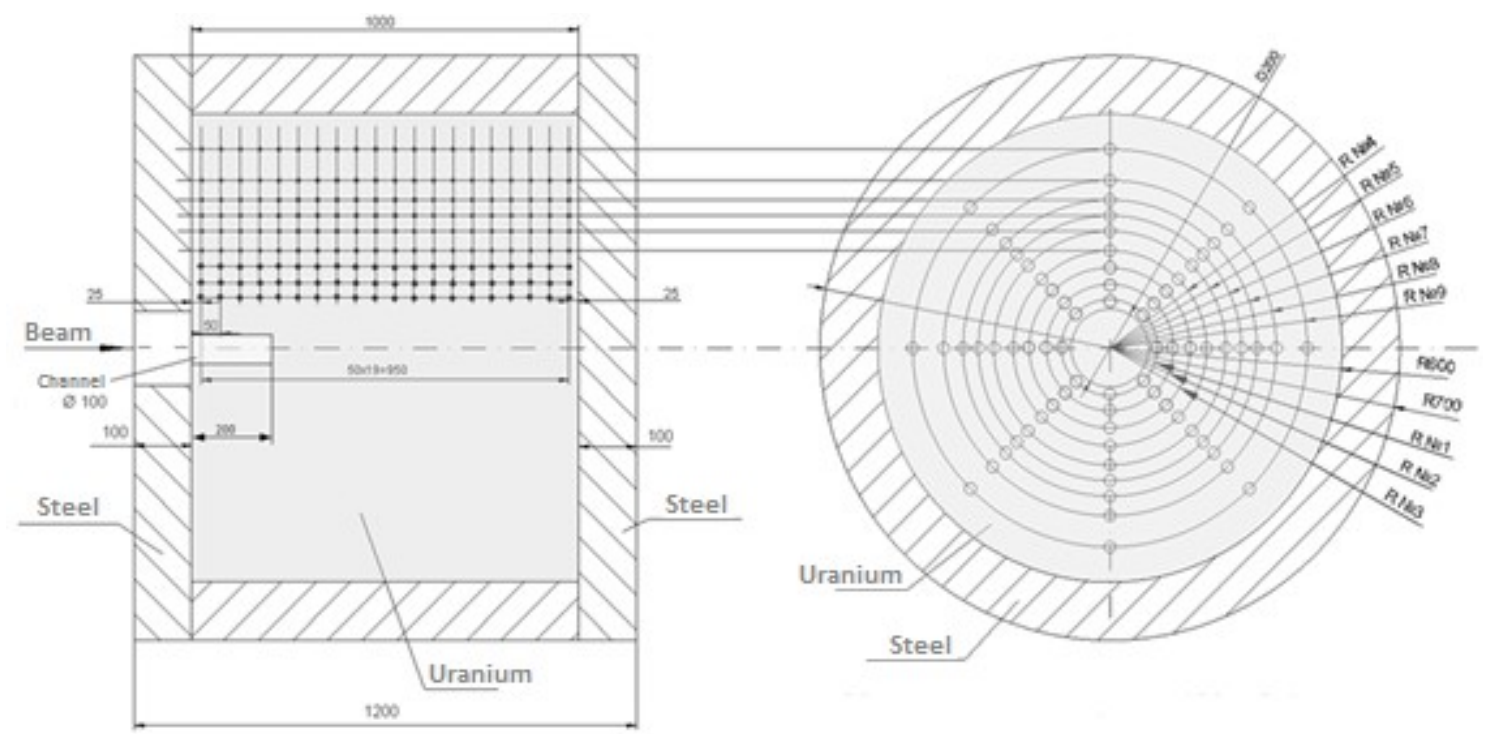

Fig. 1: Schematic drawing of Buran setup. [3]

The beam is aimed perpendicularly to the $200 \mathrm{~mm}$ in diameter opening in the front steel cover along the cylinder axis. Then it comes into the $100 \mathrm{~mm}$ in diameter channel in the uranium. This channel is supposed to have movable length of $0 \mathrm{~mm}, 100 \mathrm{~mm}$ and $200 \mathrm{~mm}$. All calculations presented in this paper were made for $200 \mathrm{~mm}$ channel length.

In the uranium blanket, there are 72 channels parallel with the central longitudinal axis. The diameter of each one is $30 \mathrm{~mm}$ and are situated uniformly along that axis in various distances from center: $140 \mathrm{~mm}, 180 \mathrm{~mm}, 220 \mathrm{~mm}, 260 \mathrm{~mm}, 300 \mathrm{~mm}, 340 \mathrm{~mm}, 380 \mathrm{~mm}, 440$ $\mathrm{mm}$ and $520 \mathrm{~mm}$.

In every channel, there are 20 measuring points for placing of detectors and transmutation samples. The first point is located in $25 \mathrm{~mm}$ distance from the uranium blanket edge. The each next point is found in $50 \mathrm{~mm}$ distance from the previous one. To sum up, there 
are 1440 measuring points in the uranium blanket. The model of Buran setup visualised in Vised $24 \mathrm{E}[4]$ can be seen in fig. 2 .

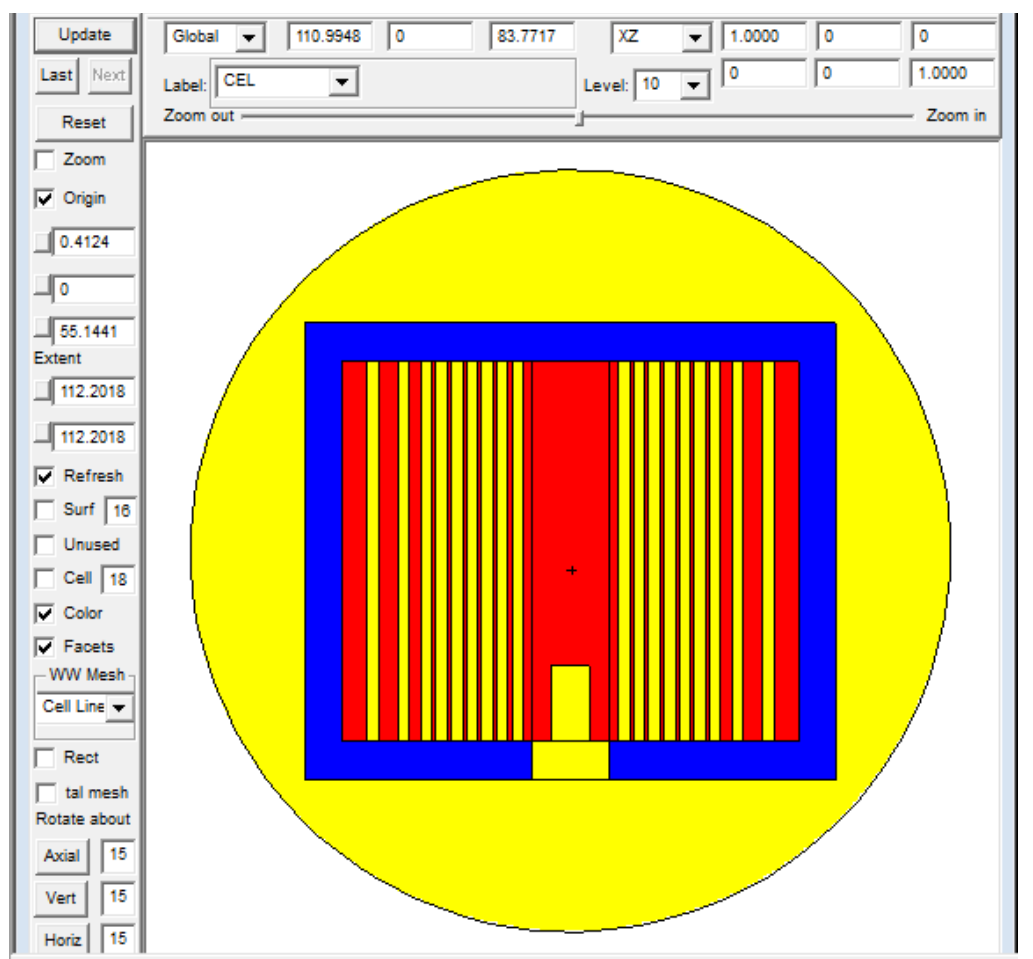

Fig. 2: Central slice of Buran mathematical model visualised in Vised editor. Red colour represents depleted uranium, blue color represents steel covering and yellow colour is air.

\subsubsection{Measuring points marking system}

For purposes of this paper, let's mark the measuring points by numbers. In view of the fact that the Buran setup can be considered as homogeneous and symmetrical, it would be useless to mark and perform calculations in all 1440 measuring points. Therefore only set of representative points was chosen. These points are apparent from fig. 3.

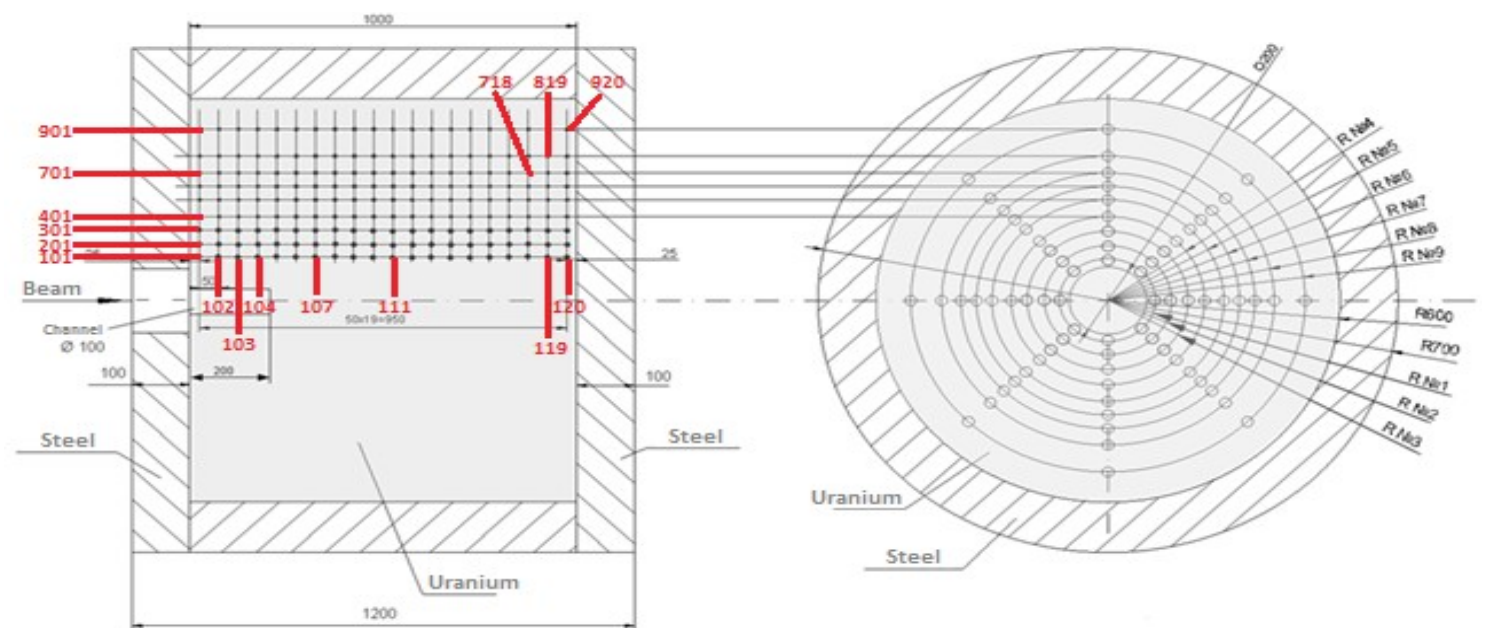

$718 \Rightarrow 7$-radial direction 18 - Iongitudinal direction

Fig. 3: System of Buran measuring points marking. 
In this figure, one can also observe the points marking. Each number related to a point consists of three digits. The first digit represents placings in radial direction while the next two digits in longitudinal direction. By term line we will understand longitudinal line. For instance, line 1 is formed by points $101-120$, line 2 by points $201-220$ etc.

\subsection{Simulations}

All simulations presented here were made for $1 \mathrm{GeV}$ proton and $1 \mathrm{GeV}$ deuteron beam incident perpendicularly on the Buran setup (see fig.1). The calculations were performed in MCNPX 2.7.0 [5] Monte Carlo code and the results were normalized to one incident particle.

Average neutron flux in the setup was investigated. Position of neutron flux maximum in every longitudinal line was determined by fitting the central part of neutron flux spectra by polynomial function of degree 6. Particular values of the longitudinal maximums were calculated by using Wolfram Alpha [6] and Polynomial \& Scientific Calculator [7].

Further, energy neutron spectra were calculated. The results are presented for three selected measuring points: 101, 106 and 515. Also neutron spectra hardening along three longitudinal lines (1,3 and 6) was investigated. In this paper, hardening in each measuring point is defined as the ratio of the total flux of neutrons with energies greater than $10 \mathrm{MeV}$ and the total flux of neutrons with energies less than or equal to $10 \mathrm{MeV}$.

Possible errors in the calculation results are negligible.

\section{Results and discussion}

\subsection{Neutron flux}

Graphs of average neutron fluxes in the Buran setup for $1 \mathrm{GeV}$ proton and $1 \mathrm{GeV}$ deuteron beam are displayed in fig. 4, 5, 6 and 7. When comparing the neutron fluxes, one can observe greater amount of neutrons formed for the deuteron beam, as was supposed.

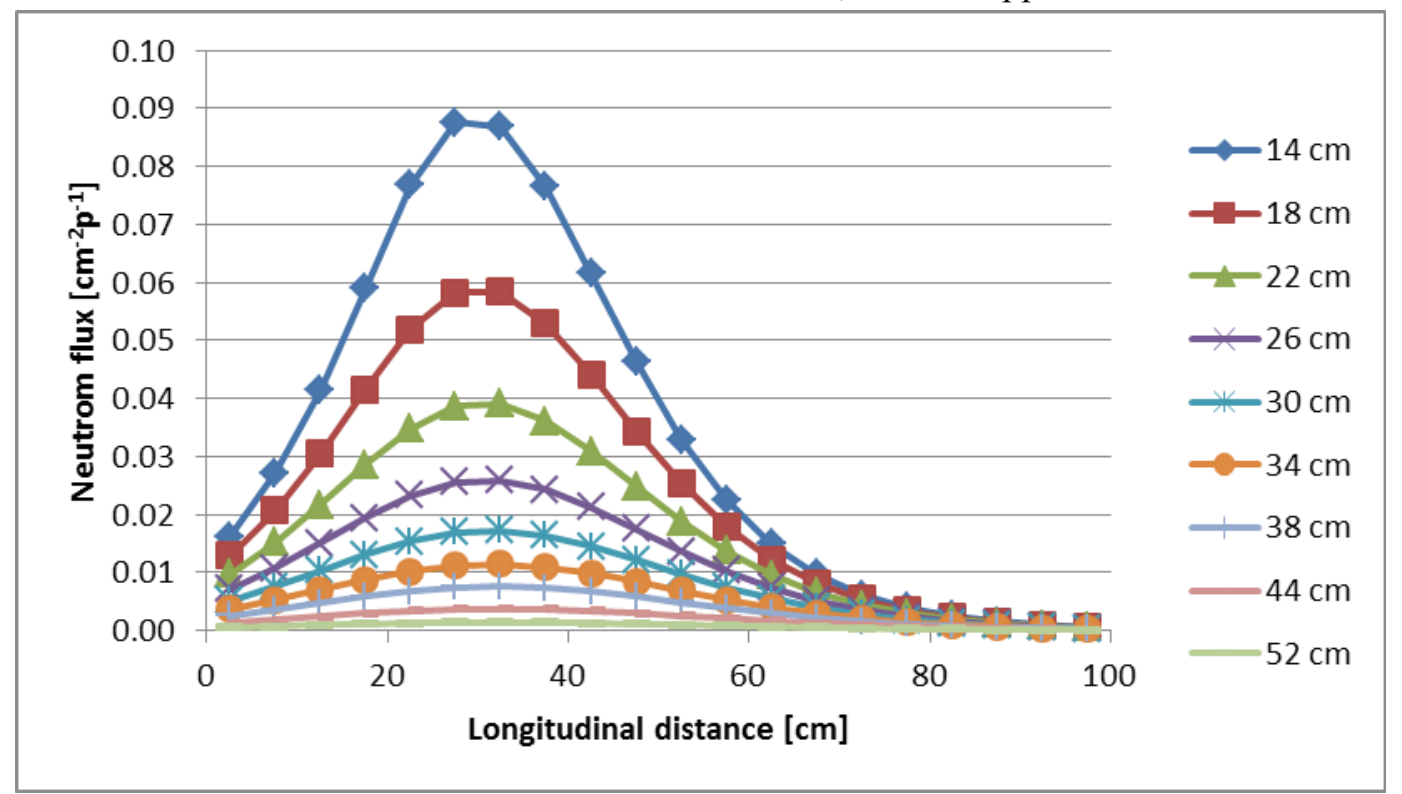

Fig. 4: Average neutron fluxes in Buran setup for $1 \mathrm{GeV}$ proton beam. Distances in the legend represent radial positions. 


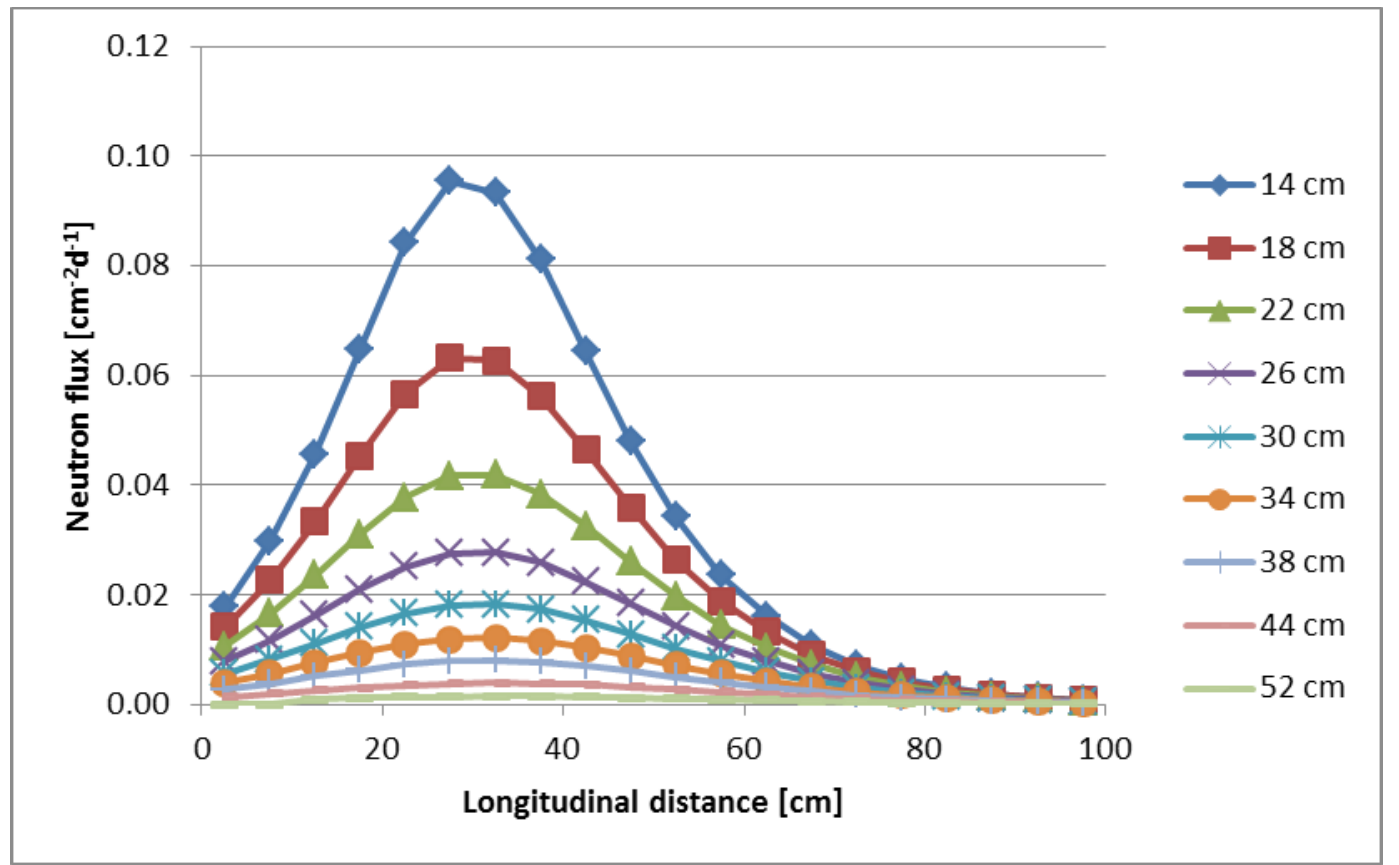

Fig. 5: Average neutron fluxes in Buran setup for $1 \mathrm{GeV}$ deuteron beam. Distances in the legend represent radial positions.

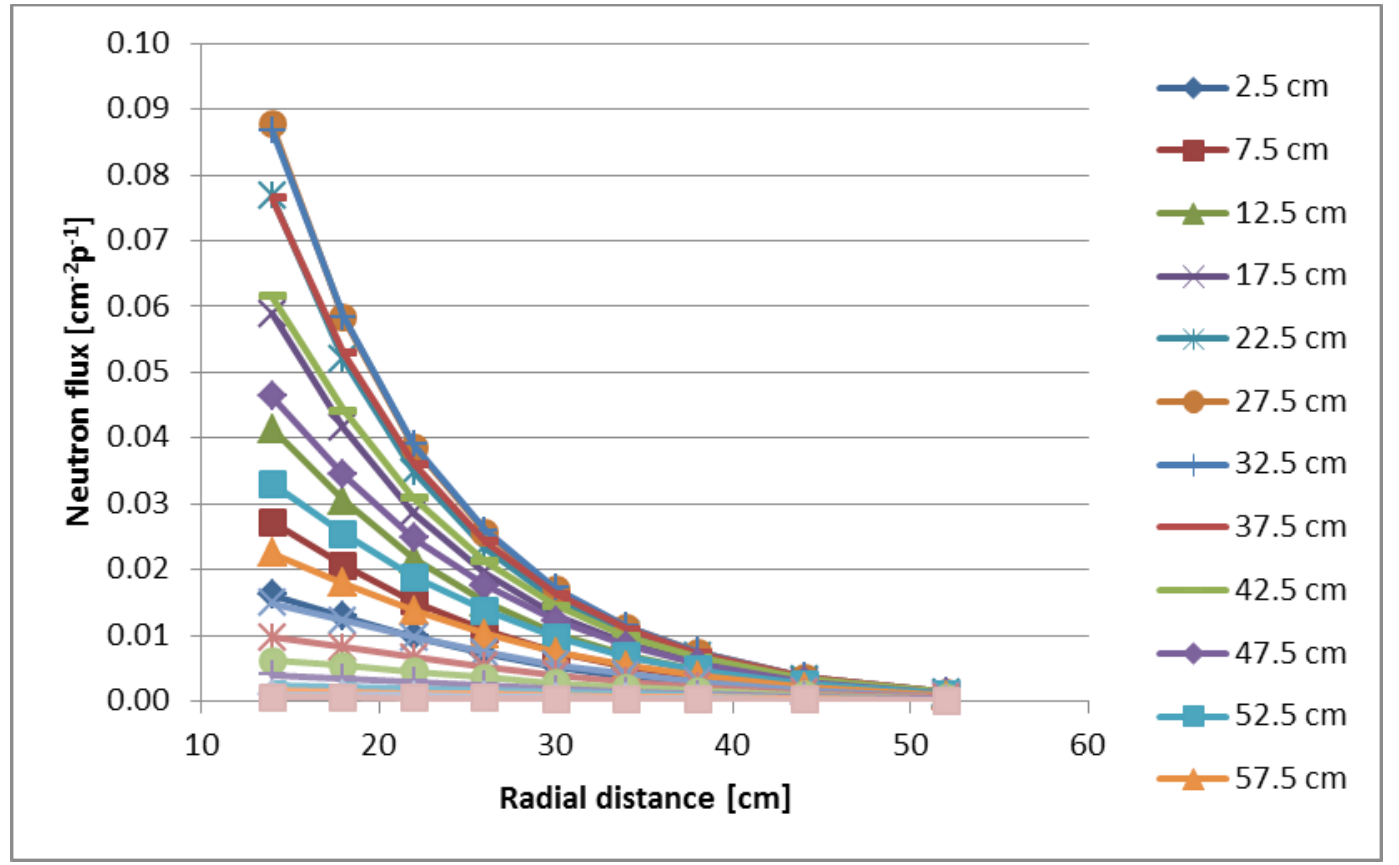

Fig. 6: Average neutron fluxes in Buran setup for $1 \mathrm{GeV}$ proton beam. Distances in the legend represent longitudinal positions. 


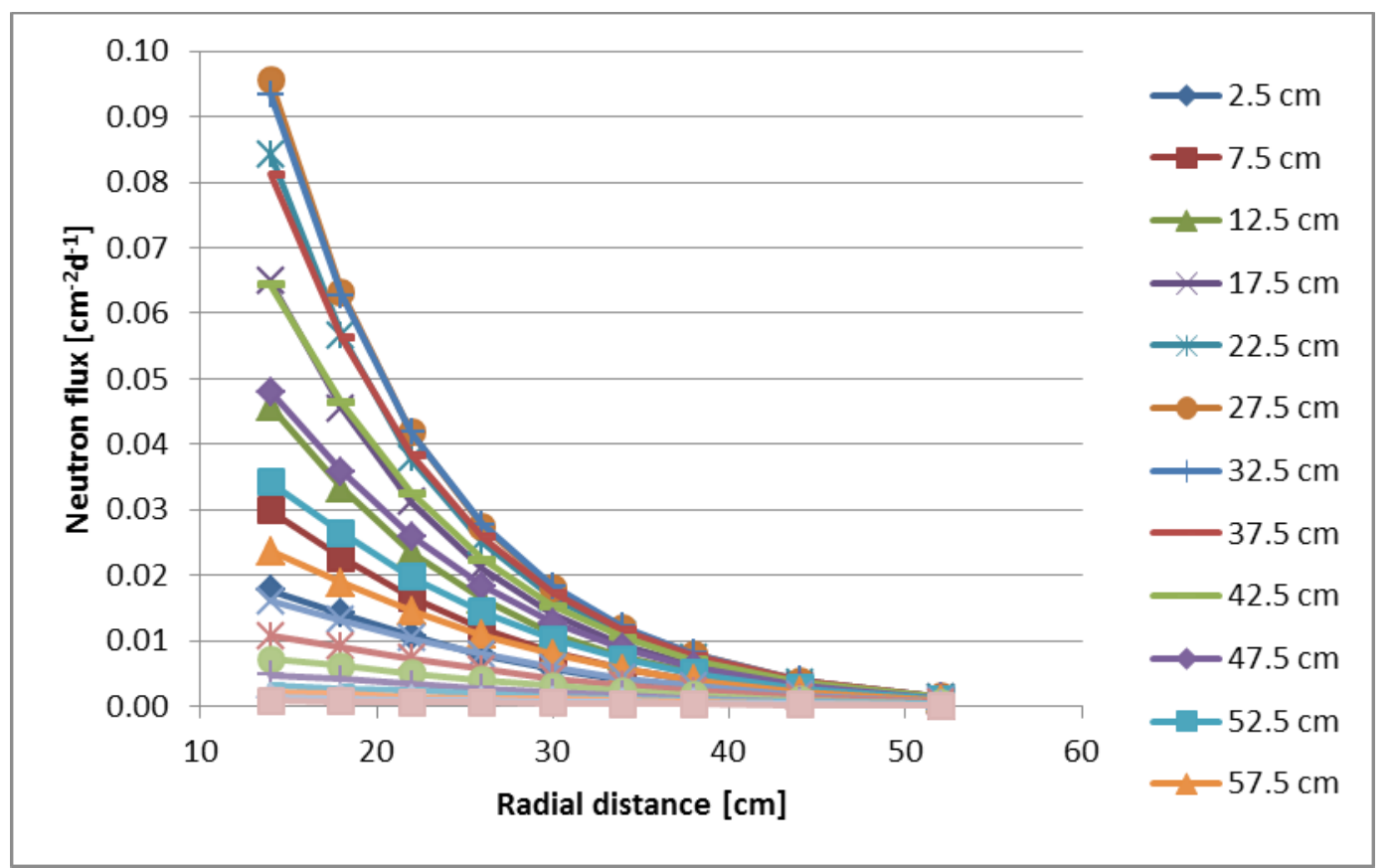

Fig. 7: Average neutron fluxes in Buran setup for $1 \mathrm{GeV}$ deuteron beam. Distances in the legend represent longitudinal positions.

The results of the neutron flux longitudinal maximums (fig. 4 and 5) investigation are visible in tab. 1 and related fig, 8. From there one can notice that the positions of the longitudinal maximums increase towards greater radial distances. Moreover, one can observe linear dependence of the maximum shifts (linear fits).

\begin{tabular}{|r|r|r|}
\hline $\begin{array}{l}\text { Radial distance } \\
{[\mathrm{cm}]}\end{array}$ & $\begin{array}{l}\text { Maximums for 1GeV proton beam } \\
{[\mathrm{cm}]}\end{array}$ & $\begin{array}{l}\text { Maximums for 1GeV deuteron beam } \\
{[\mathrm{cm}]}\end{array}$ \\
\hline $14 \mathrm{~cm}$ & 29.73 & 29.28 \\
\hline $18 \mathrm{~cm}$ & 30.13 & 29.69 \\
\hline $22 \mathrm{~cm}$ & 30.53 & 30.13 \\
\hline $26 \mathrm{~cm}$ & 30.79 & 30.53 \\
\hline $30 \mathrm{~cm}$ & 31.25 & 30.92 \\
\hline $34 \mathrm{~cm}$ & 31.60 & 31.37 \\
\hline $38 \mathrm{~cm}$ & 32.01 & 31.76 \\
\hline $44 \mathrm{~cm}$ & 32.61 & 32.55 \\
\hline $52 \mathrm{~cm}$ & 33.37 & 33.44 \\
\hline
\end{tabular}

Tab. 1: Positions of neutron flux longitudinal maximums (calculated from the polynomial function fits) for $1 \mathrm{GeV}$ proton and $1 \mathrm{GeV}$ deuteron beam. 


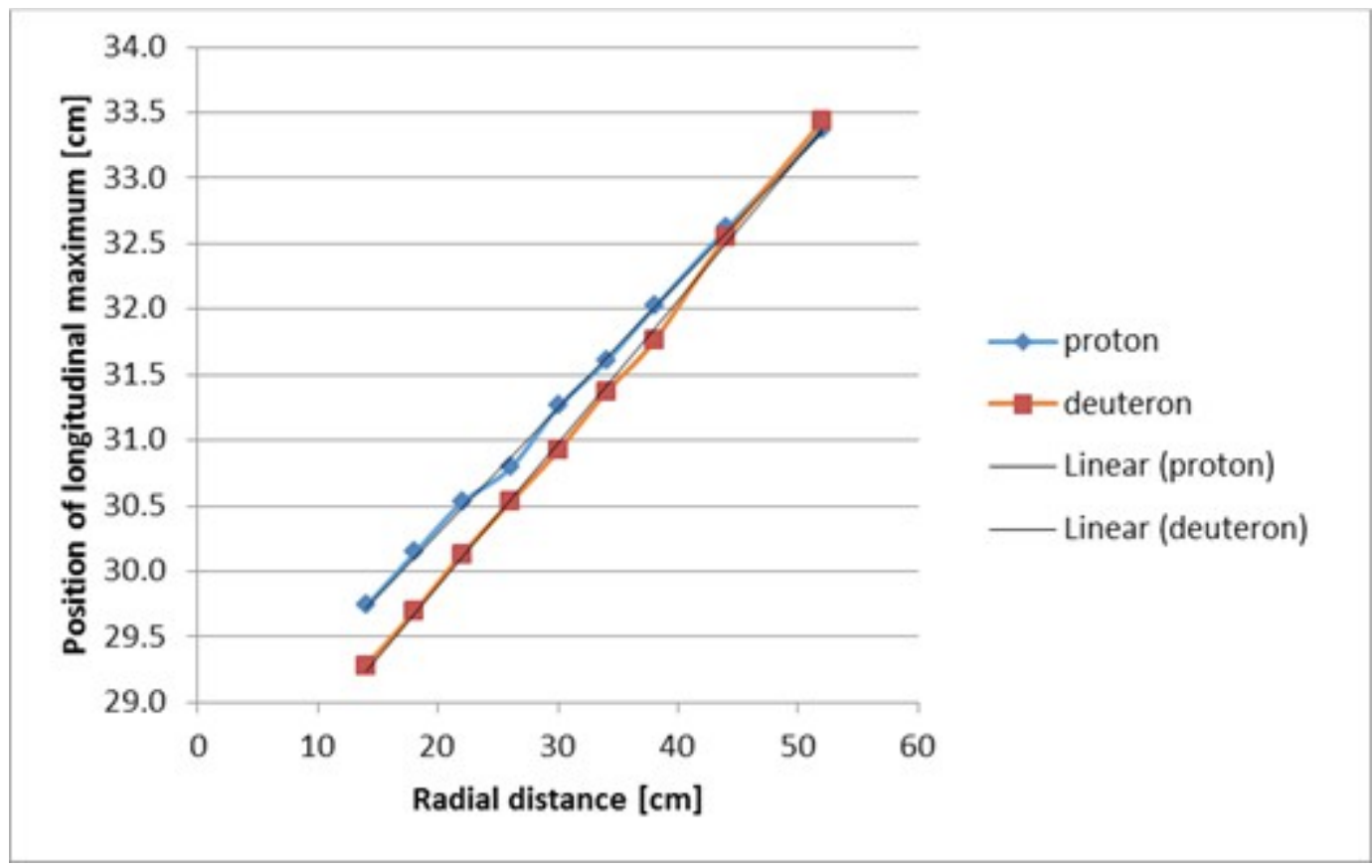

Fig. 8: Longitudinal maximums in dependence on radial distance. Marks are corresponding to data from tab. 1 .

\subsection{Energy neutron spectra}

Energy neutron spectra for $1 \mathrm{GeV}$ proton and $1 \mathrm{GeV}$ deuteron beam are displayed in fig. 9 and 10. One can see that the graphs for proton and deuteron beam are of similar shape. As supposed, values of neutrons produced in the spectra for deuteron beam are greater than in the spectra for proton beam.

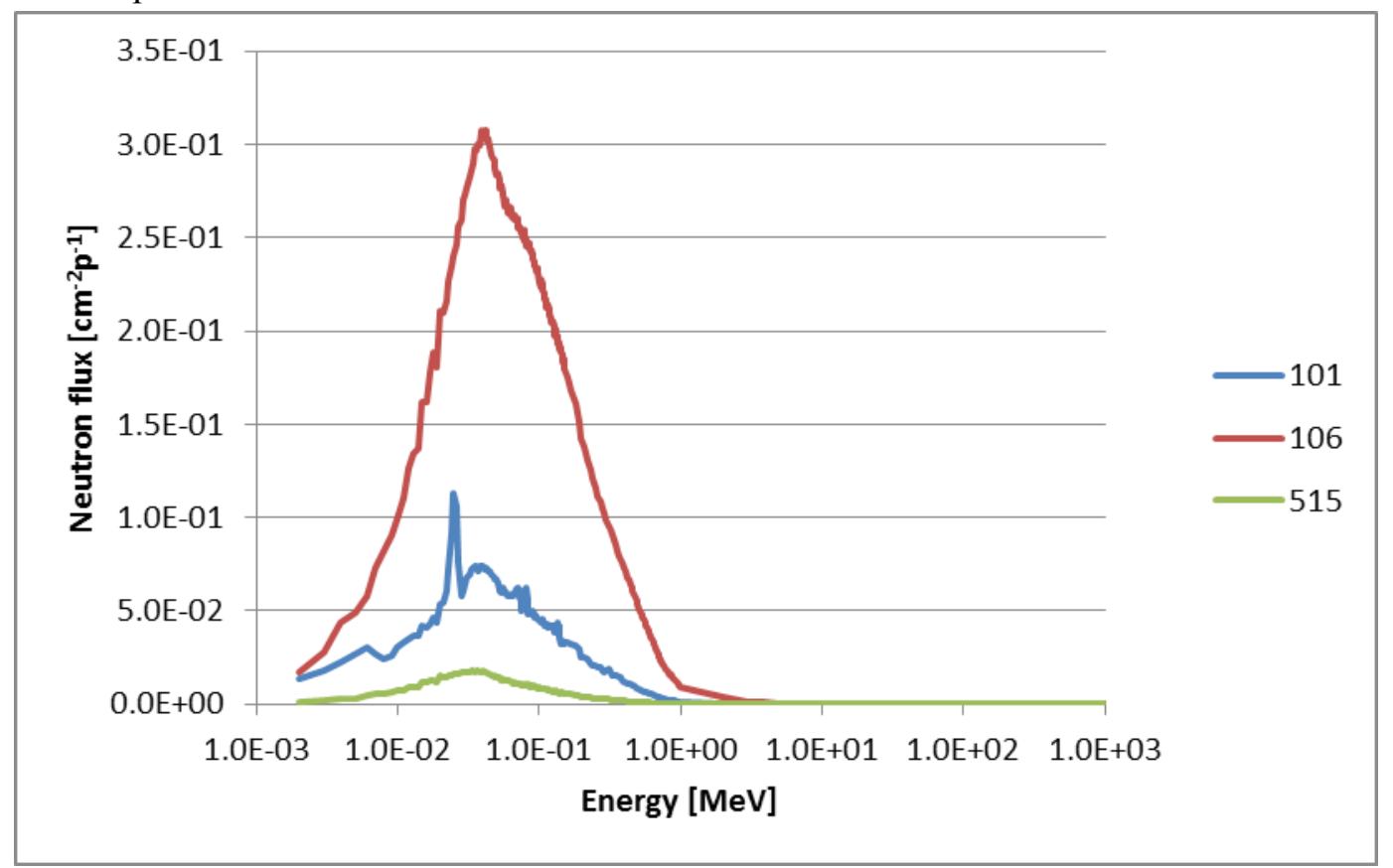

Fig. 9: Energy neutron spectra for $1 \mathrm{GeV}$ proton beam in representative measuring points 101, 106 and 515 . 


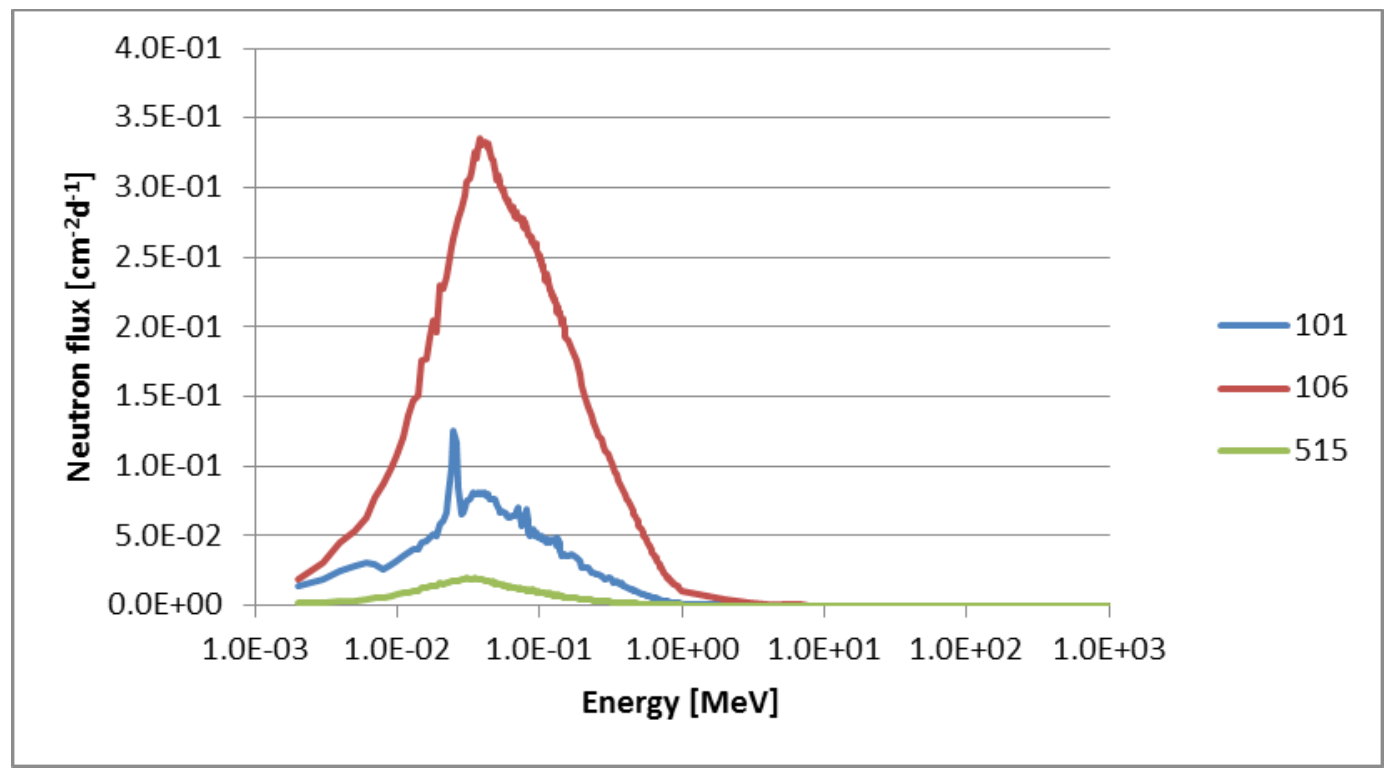

Fig. 10: Energy neutron spectra for $1 \mathrm{GeV}$ deuteron beam in representative measuring points 101,106 and 515 .

Dependences of the neutron spectra hardening on longitudinal distance are displayed in fig. 11 and 12. At lower distances, there are not visible significant differences in hardness both for proton and deuteron beam. But at higher distances, greater values of hardness in spectra where deuteron beam was used can be found. Further, the curves cross each other at longitudinal distances of about 14 and $60 \mathrm{~cm}$. It can be concluded that around these values, the hardness is approximately the same for all radial distances. Furthermore, one can notice that the shape of dependences becomes more linear with increasing radial distance. This, in fact, confirms that the most remote distances are reached by the most energetic neutrons.

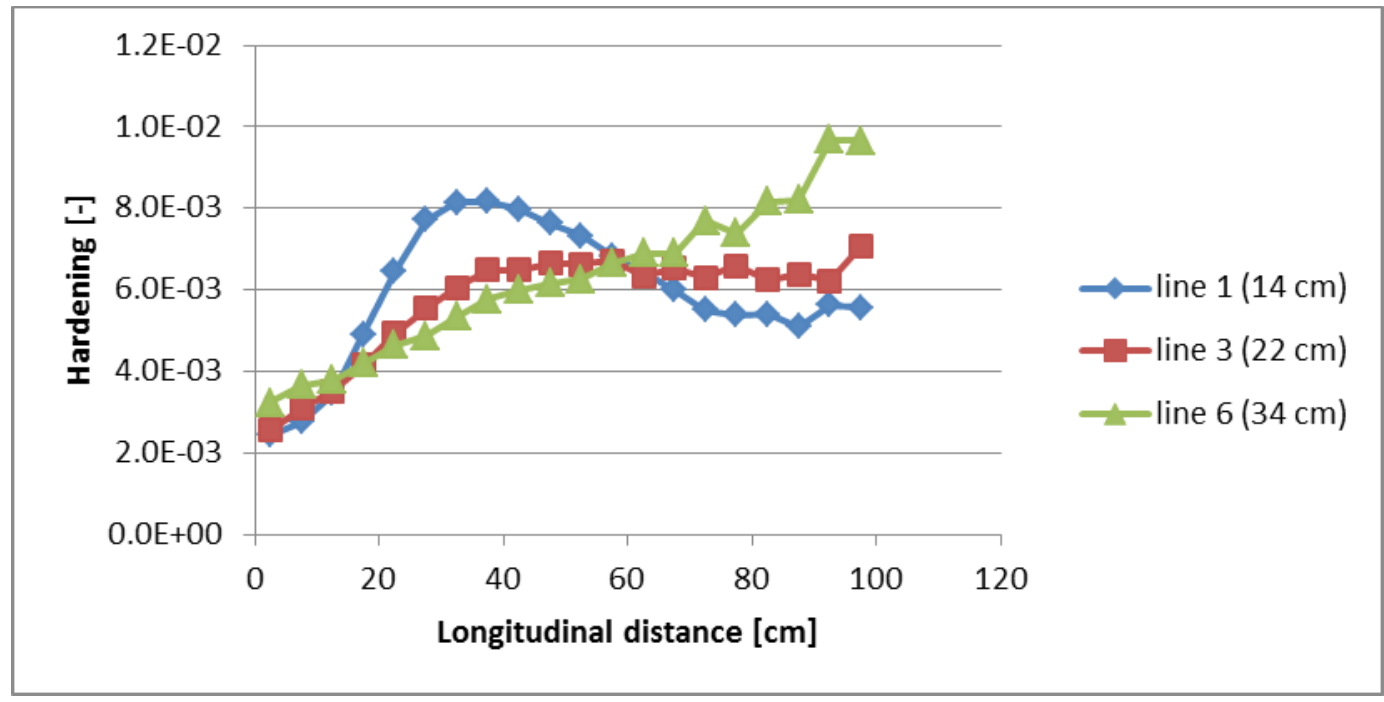

Fig. 11: Neutron spectra hardening in dependence on longitudinal distance for $1 \mathrm{GeV}$ proton beam. Hardening is displayed for three longitudinal lines. Distances in the brackets mean radial positions of the lines. 


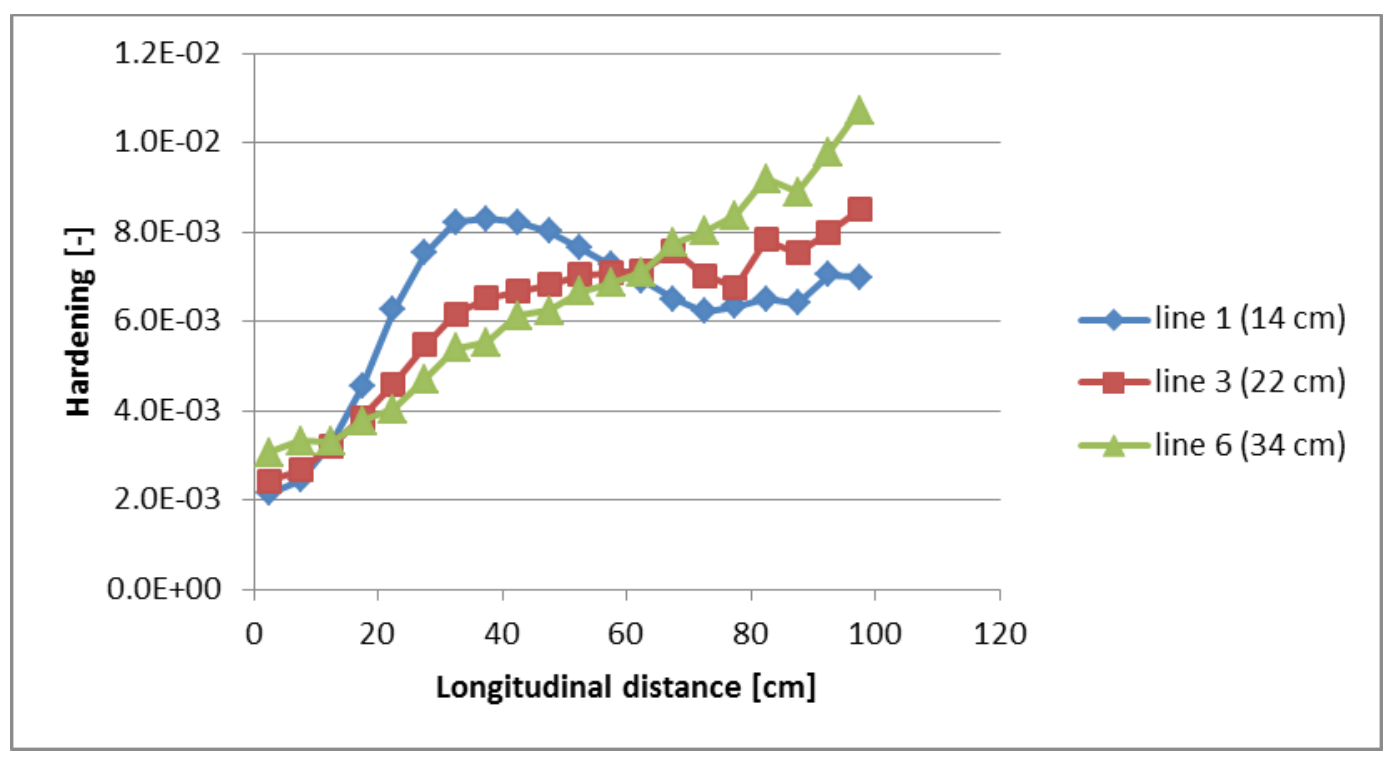

Fig. 12: Neutron spectra hardening in dependence on longitudinal distance for $1 \mathrm{GeV}$ deuteron beam. Hardening is displayed for three longitudinal lines. Distances in the brackets mean radial positions of the lines.

\section{Conclusions}

Some characteristics of neutron production and transport through the new Buran setup irradiated with $1 \mathrm{GeV}$ proton and $1 \mathrm{GeV}$ deuteron beam were calculated (using mainly MCNPX 2.7.0 code), presented and discussed. We focused on average neutron fluxes and positions of their longitudinal maximums in the setup and also on energy neutron spectra and their hardening. The most surprising results found are that the positions of the neutron flux longitudinal maximums increase linearly towards greater radial distances and that the hardness of energy neutron spectra is approximately the same for all radial distances around longitudinal distance of 14 and $60 \mathrm{~cm}$ (both for proton and deuteron beam).

\section{References}

[1] P. Chudoba, Využití aktivačnich detektorů při měrení neutronového pole v modelových sestavách ADTS, Master Thesis, Charles University, Prague 2013.

[2] O. Svoboda, Experimental Study of Neutron Production and Transport for ADTT, PhD Thesis, Czech Technical University in Prague, Prague 2011.

[3] Documents of collaboration E\&T RAW.

[4] A. L. Schwarz, R. A. Schwarz, L. L. Carter, MCNP/MCNPX Visual Editor Computer Code Manual for Vised Version 24E, 2011.

[5] D. B. Pelowitz, MCNPX User's Manual Version 2.7.0, Los Alamos National Laboratory, 2011.

[6] Wolfram Alpha, [online at http://wolframalpha.com].

[7] Polynomial \& Scientific Calculator, [online at http://xrjunque.nom.es/precis/polycalc.aspx]. 